\title{
Testing the National Alcohol Policy Score Card (NAPSC) to assess progress in implementing a comprehensive policy response to reduce the harmful use of alcohol in South Africa
}

\author{
Charles D. Parry \\ Alcohol \& Drug Abuse Research Unit, South African Medical Research Council and Department of Psychiatry, University of Stellenbosch, Cape \\ Town, South Africa
}

\begin{abstract}
Aims: To complement recent alcohol policy initiatives of WHO, a study was designed to test the feasibility of a simple instrument to assess the state of alcohol policy development and implementation in a developing country.

Design: A cross-sectional survey.

Setting: Data were collected via a web-survey.

Participants: 52 experts across various sectors were approached.

Measures: Study participants were asked to complete a 13-item web survey that draws on the target areas for national action identified in the World Health Organization (WHO) Global Strategy to Reduce the Harmful Use of Alcohol (2010). The state of policy development and implementation was assessed for 2011 and then retrospectively to 2006. Participants were also asked to comment on the ease of completing the survey.
\end{abstract}

Findings: Based on the responses from 37 experts, improvements were noted in alcohol policy development and implementation in all areas over time, with particular movement in developing a national alcohol strategy; increasing leadership, awareness and commitment; drink-driving; and health services' response. The total (average) score of 35\% in 2011, while up by 11 percentage points, indicates that much work remains to be done, particularly to restrict the marketing of alcoholic beverages, address informal alcohol, increase community action to address harmful alcohol use, and increase financial resources.

Conclusions: Participants found the web-survey easy to use. The overall findings and the way they are presented could be used to promote discussions around the development and implementation of national alcohol strategies and how they change over time, and even to compare the situations in different countries. Refinement of the instrument continues.

In May 2010 the Global Strategy to Reduce the Harmful Use of Alcohol was approved by the World Health Assembly (WHA). A major goal of the Strategy is to support and complement public health policies in member states. Within the Strategy, policy options and interventions available for national action are grouped into 10 recommended target areas: (1) leadership, awareness and commitment, (2) health services' responses, (3) community action, (4) drink-driving policies and countermeasures, (5) availability of alcohol, (6) marketing of alcoholic beverages, (7) pricing policies, (8) reducing the negative consequences of drinking and alcohol intoxication, (9) reducing the public health impact of illicit and informally produced alcohol, and (10) monitoring and surveillance (World Health Organization, 2010 [WHO]). The Strategy, however, acknowledges that not all policy options and interventions will be applicable or relevant for all member states. It furthermore indicates that implementation of the Strategy by member states requires appropriate mechanisms at various levels for assessment, reporting and reprogramming. However, it gives little guidance on how countries should carry this out.

Since the 1980s, various tools have been devised to measure alcohol control policies. For example, Davies and Walsh (1983) developed a 30-item scale which was used to rank countries according to the degree of alcohol control. These scales covered four domains: "control of production," "control of distribution," "social (and environmental) measures," and "(price and) fiscal measures.” Anderson and Lehto (1995) further developed this scale. Their instrument covered policy areas such as 
advertising bans, restrictions on the maximum alcohol content of beverages, warning labels on alcoholic beverages, and random breath testing. In addition, they attempted to measure the differences in enforcement of alcohol control legislation, but only in the areas of controls on production and distribution of alcohol. Hilton and Johnstone (1988) developed a scale comprising 16-items that asked about the presence or absence of specific alcohol-related control measures, but, again, did not measure the extent of implementation.

Later Karlsson and Österberg (2001) developed the 14-item European Comparative Alcohol Study (ECAS) Scale, which was based on the earlier alcohol control scales but omitted questions about alcohol taxation. It was divided into six domains: control of production and wholesale, control of distribution, personal control, control of marketing, social and environmental controls, and public policy. It also focused on the policies existing in the different countries, rather than the extent to which these policies were implemented. This was followed by the Alcohol Policy Index (Brand, Saisana, Rynn, Pennoni, \& Lowenfels, 2007). This 16-item scale generates a score based on policies from five regulatory domains: physical availability of alcohol, drinking context, alcohol prices, alcohol advertising, and operation of motor vehicles. Different weights are assigned to each item based on the perceived effectiveness of regulations aimed at reducing the adverse effects of alcohol (Babor et al., 2010). This scale also did not attempt to measure the enforcement or implementation of these policies.

While pure alcohol consumption in South Africa, at 9.5 litres per annum per capita, is not high, when the high levels of abstention are taken into account, this country has one of the highest levels of per capita consumption per drinker globally. It was also identified as having one of the highest levels of heavy episodic drinking globally among men and women, and one of the most negative ratings in Africa on an index measuring harmful patterns of drinking (World Health Organization, 2011). Since the country's first democratic government was elected in 1994, there have been shifts in alcohol policy in areas such as excise taxes, restrictions on allowable packaging for alcohol products, maximum allowable blood alcohol concentration levels for drivers, and the imposition of warning labels on alcohol containers (Parry, 2010). More recently, the government has articulated the need for stronger action to be taken to reduce harmful use of alcohol, including calling for increasing the legal drinking/purchase age to 21 years, making any drinking and driving illegal, and banning all alcohol advertising (Department of Social Development, 2011).

The purpose of this study is to test the feasibility of, and analyse responses to, a simple instrument designed to measure both the state of alcohol policy development and the degree of its implementation, at two points in time, in a developing country which has high levels of harmful alcohol use and which has seen recent changes in terms of alcohol policy development and implementation.

\section{Method}

\section{Design}

A cross-sectional descriptive study was undertaken.

\section{Sampling}

Persons identified by the author as having a broad level of expertise in alcohol policy across South Africa from government, academic/research, non-governmental and community-based organisations (NGOs/CBOs) were identified and included in the study sample. The author has been involved in alcohol policy work in South Africa for over 20 years and has a broad network of contacts in all of these sectors.

\section{Measurements}

The National Alcohol Policy Score Card (NAPSC), a 12item self-report instrument for assessing progress in terms of the development and implementation of policy responses to reduce harmful use of alcohol, was developed. It draws on the 10 target areas for national action referred to above (World Health Organization, 2010), with two additional items (1) whether a country has a stand-alone national alcohol strategy, and (2) whether such a national alcohol strategy is adequately financed (Table 1). The person completing the web-survey was also asked for information on the sector that best reflected his or her main area of work, and for a brief comment on the ease or difficulty of completing the survey. Given the sample, the instrument was available only in English. An example of two items on the NAPSC is given in Appendix 1.

Participants were required to give a response on a scale of 1 to 10 for each of the 12 items, reflecting what they believed to be the current situation (i.e., in 2011) in South Africa. The opposing endpoints of the scale represented extreme positions (e.g., 1 = "No national policy or very fragmented" versus $10=$ "Well-set-out, stand-alone policy with short-, medium-, and long-term measurable objectives based on good evidence”), based upon the principal investigator's view of what would constitute extreme positions for that item. Definitions of the endpoints for each of the 12 areas were provided (with an additional definition for a response of 9 on the item dealing with drink-driving policies and countermeasures).

\section{Procedures}

On August 25, 2011, study participants were sent an e-mail inviting them to participate in a web survey to assess the state of alcohol policy development and implementation in South Africa currently (in 2011) and five years previously (in 2006). They were informed that it would take 15 to 20 minutes to complete. Prior to participating in the web survey, participants were informed about the study and asked to give their consent to participate. They were informed that their anonymity would be preserved. Ethical approval for the study was granted by the University of Stellenbosch. Two reminders were sent. 
Table 1

12 NAPSC items

\begin{tabular}{lll}
\hline$\#$ & Item & Short name \\
\hline 1 & National alcohol strategy & Alcohol strategy \\
2 & Leadership, awareness \& commitment & Leadership \\
3 & Pricing (excise tax) & Pricing \\
4 & Marketing of alcoholic beverages & Marketing \\
5 & Availability of alcohol & Availability \\
6 & Health services response & Health services \\
7 & Drink-driving policies \& countermeasures & Drink driving \\
8 & Community action & Community action \\
9 & Reducing negative consequences of drinking \& intoxication & Harm reduction \\
10 & Reducing public health impact of illicit alcohol \& informally produced alcohol & Informal alcohol \\
11 & Monitoring \& surveillance & Monitoring \& surveillance \\
12 & Financial resourcing & Financing \\
\hline
\end{tabular}

\section{Data analysis}

The data are represented primarily via descriptive statistics, and also graphically via a radar chart. Total ratings for each participant on each of the 12 items assessing policy development and implementation for each of the two time periods were also calculated. These have been weighted (i.e., multiplied by 0.833 ) to yield a rating between 10 and 100 .

\section{Results}

\section{Responses to the request to participate}

A total of 37 of the 52 experts approached responded to the survey $(71.2 \%)$. As the number identifying their sector as "treatment" or "other" were very low (1 each), and as none of the experts approached from treatment centres were government employees, respondents from these sectors were grouped with those from NGOs and CBOs, yielding six experts from the government sector (out of a possible 12), 13 from the academic/research sector (out of a possible 19), and 18 from the re-formulated NGO/CBO sector (out of a possible 21).

\section{Comparison of ratings across the 12 items and comparisons across time}

The minimum, maximum, mean, standard deviation, skewness, median, and mode for ratings on each of the NAPSC items for 2011 and 2006 are indicated in Table 2. The ratings varied widely; ratings on some items ranged from 1 to 10, while others had a much narrower range. All but one of the items were positively skewed. Given the nature of the data, it was decided to look at the rank ordering of mean scores across the different items as they pertain to the alcohol policy situation at the two points in time (Table 3). A low ranking indicates a higher mean rating (i.e., a more advanced level of policy development and implementation). At both points in time, items like
Drink driving and Pricing had lower rankings than other items, and items such as Financing, Informal alcohol, and Community action were consistently ranked higher than other items. Some items showed positive movement over the five years in terms of their ranking (e.g., Leadership), whereas others showed a decline over time (e.g., Marketing).

The comparison of mean ratings on the 10-point scale of the different items at 2010 and 2006, as well as over the five years, is demonstrated graphically in Figure 1. Improvements in alcohol policy development and implementation in all areas were noted over time, with particular movement in the areas of developing a national alcohol strategy; increasing leadership, awareness and commitment; health services' response; and drink-driving. The total (average) rating of 35\% showed an increase of 11 percentage points over the five-year period (from 24\%). There appears to be little movement in the ratings on the following items over time: marketing, availability, informal alcohol, and financing.

\section{Comparison of ratings across sectors}

There was not much difference between the different sectors on average total ratings (across the 12 items) in 2011 and 2006, with respondents from the government sector, academic/research sector and NGO/CBO sectors having average total ratings of $36.7 \%, 36.4 \%$, and $32.9 \%$ in 2011 respectively, compared to $22.6 \%, 25.5 \%$ and $22.8 \%$ respectively in 2006. However, when ratings of the 12 individual items in the NAPSC in 2011 are compared across sectors, there do appear to be differences (Table 4). In particular, respondents from the government sector appear to rate Leadership much higher than the other two sectors, and Availability higher than respondents from the NGO/CBO sector. In contrast, academics appear to rate policy development and implementation in the Marketing 
Table 2

Minimum, maximum, mean, standard deviation, skewness, median, and mode for ratings on the 12 NAPSC items and total ratings (weighted) for 2011 and 2006

\begin{tabular}{|c|c|c|c|c|c|c|c|c|}
\hline$\#$ & Item & Minimum & Maximum & Mean & $S D$ & Skewness & Median & Mode \\
\hline \multicolumn{9}{|c|}{2011} \\
\hline 1 & Alcohol strategy & 1 & 7 & 3.65 & 1.92 & 0.06 & 4 & 1 \\
\hline 2 & Leadership & 1 & 10 & 4.27 & 2.54 & 0.35 & 4 & 1,6 \\
\hline 3 & Pricing & 1 & 10 & 4.27 & 2.28 & 0.23 & 4 & 4 \\
\hline 4 & Marketing & 1 & 10 & 3.14 & 2.12 & 1.34 & 3 & 1 \\
\hline 5 & Availability & 1 & 10 & 3.32 & 2.20 & 0.97 & 3 & 1 \\
\hline 6 & Health services & 1 & 9 & 3.84 & 2.30 & 0.69 & 3 & 3 \\
\hline 7 & Drink driving & 1 & 10 & 4.59 & 2.17 & 0.45 & 4 & 6 \\
\hline 8 & Community action & 1 & 6 & 3.05 & 1.83 & 0.44 & 3 & 1 \\
\hline 9 & Harm reduction & 1 & 7 & 3.19 & 1.65 & 0.11 & 3 & 5 \\
\hline 10 & Informal alcohol & 1 & 6 & 2.54 & 1.57 & 0.78 & 2 & 1 \\
\hline 11 & Monitoring \& surveillance & 1 & 9 & 3.51 & 1.88 & 0.65 & 3 & 3,5 \\
\hline \multirow[t]{2}{*}{12} & Financing & 1 & 5 & 2.32 & 1.23 & 0.67 & 2 & 2 \\
\hline & Total rating & & 55.8 & 34.75 & 11.63 & -0.10 & 35.0 & 35 \\
\hline \multicolumn{9}{|c|}{2006} \\
\hline 1 & Alcohol strategy & 1 & 5 & 2.19 & 1.29 & 0.78 & 2 & 1 \\
\hline 2 & Leadership & 1 & 5 & 2.16 & 1.14 & 0.73 & 2 & 1 \\
\hline 3 & Pricing & 1 & 7 & 3.16 & 1.83 & 0.55 & 3 & 4 \\
\hline 4 & Marketing & 1 & 8 & 2.43 & 1.73 & 1.48 & 2 & 1 \\
\hline 5 & Availability & 1 & 10 & 2.78 & 1.87 & 1.73 & 2 & 1 \\
\hline 6 & Health services & 1 & 5 & 2.35 & 1.25 & 0.63 & 2 & 2 \\
\hline 7 & Drink driving & 1 & 10 & 3.16 & 2.05 & 1.33 & 3 & 2 \\
\hline 8 & Community action & 1 & 5 & 2.00 & 1.25 & 1.00 & 1 & 1 \\
\hline 9 & Harm reduction & 1 & 5 & 2.24 & 1.34 & 0.84 & 2 & 1 \\
\hline 10 & Informal alcohol & 1 & 5 & 1.95 & 1.08 & 0.95 & 2 & 1 \\
\hline 11 & Monitoring \& surveillance & 1 & 5 & 2.32 & 1.29 & 0.58 & 2 & 1 \\
\hline \multirow[t]{2}{*}{12} & Financing & 1 & 4 & 1.73 & 0.93 & 1.24 & 1 & 1 \\
\hline & Total rating & & 47.5 & 23.74 & 8.97 & 0.52 & 23.3 & 31.7 \\
\hline
\end{tabular}

Table 3

Rank ordering of the mean ratings on the various items at 2011 and 2006

\begin{tabular}{lcc}
\hline Item & $\mathbf{2 0 1 1}$ & $\mathbf{2 0 0 6}$ \\
\hline Alcohol strategy & 5 & 8 \\
Leadership & 2 & 9 \\
Pricing & 2 & 1 \\
Marketing & 9 & 4 \\
Availability & 7 & 3 \\
Health services & 4 & 5 \\
Drink driving & 1 & 1 \\
Community action & 10 & 10 \\
Harm reduction & 8 & 7 \\
Informal alcohol & 11 & 11 \\
Monitoring \& surveillance & 6 & 6 \\
Financing & 12 & 12 \\
\hline
\end{tabular}

A low ranking implies a higher level of policy development and implementation 
Figure 1

Comparison of the mean ratings on the items of the NAPSC in 2006 and 2011

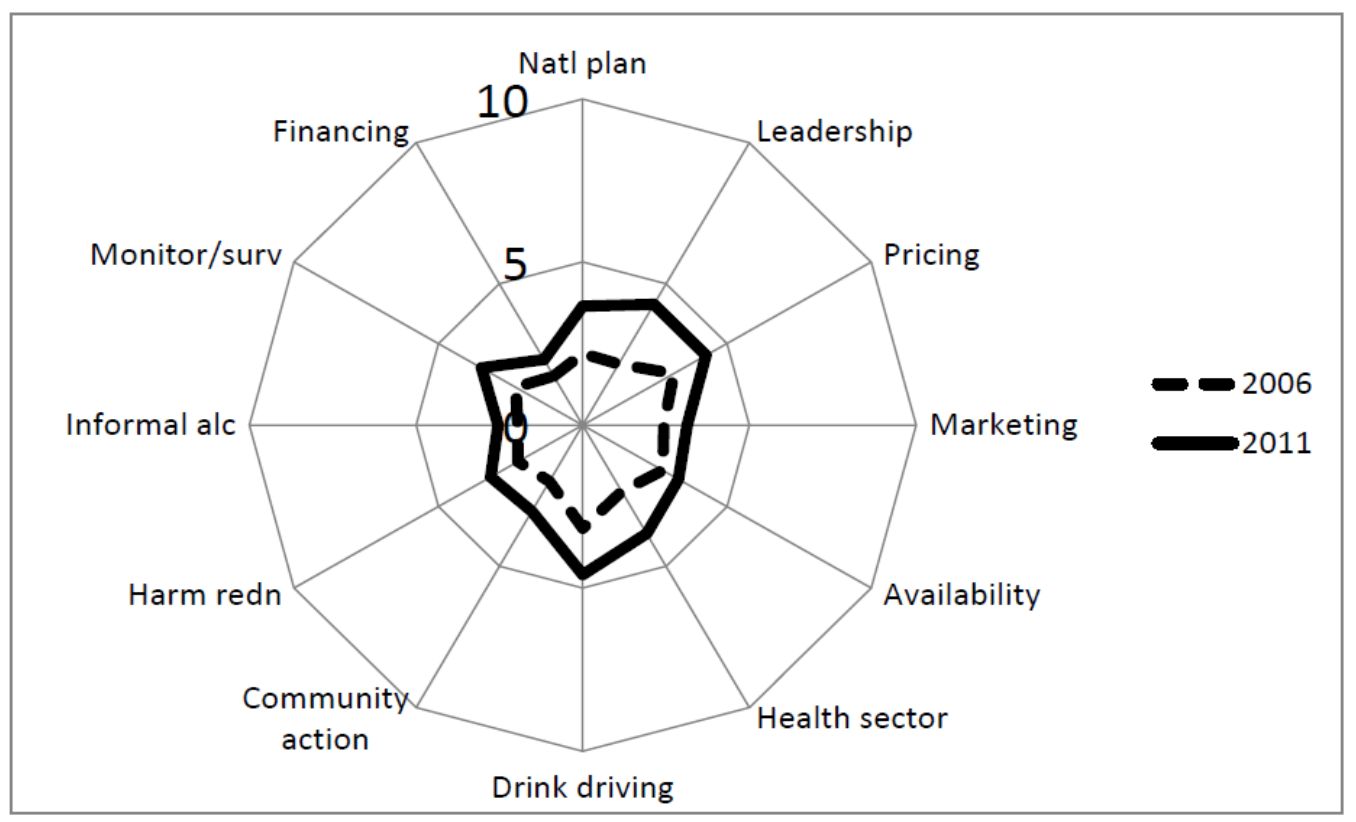

Table 4

Comparison of mean ratings across sectors on the NAPSC in 2011 (out of 10)

\begin{tabular}{lccc}
\hline & \multicolumn{2}{c}{ Sector } \\
\cline { 2 - 4 } Item & Government & Academic/research & NGO/CBO \\
\hline Alcohol strategy & 3.7 & 4.3 & 3.2 \\
Leadership & 6.0 & 3.9 & 3.9 \\
Pricing & 3.0 & 4.4 & 4.6 \\
Marketing & 2.2 & 3.9 & 2.9 \\
Availability & 4.5 & 4.3 & 2.2 \\
Health services & 4.2 & 3.9 & 3.7 \\
Drink driving & 5.8 & 4.5 & 4.2 \\
Community action & 2.7 & 2.8 & 3.4 \\
Harm reduction & 3.3 & 3.1 & 3.2 \\
Informal alcohol & 2.3 & 2.8 & 2.4 \\
Monitoring \& surveillance & 3.7 & 3.4 & 3.6 \\
Financing & 2.7 & 2.5 & 2.1 \\
\hline
\end{tabular}

area substantially higher than the respondents from the government sector.

\section{Ease/difficulty of completing the survey and suggestions for improvement}

Comments were received from 17 respondents on this item. Two-thirds were positive ("clear, user friendly"; "easy to complete"; "great survey format—did it on my cell phone"; "examples of interventions/policy measures facilitate decision-making”; “a very thought-provoking task, thanks"). Of the five negative comments or comments suggesting change, one was very minor ("transpose the two years in the web survey"), whereas others indicated that the respondents did not feel qualified to answer all items, or felt that they needed to be provided with more information to assist them in making their rating $(n=3)$. One indicated 
that the task was too complex and that it would be better to break the items into sub-items.

\section{Discussion}

Most alcohol policy surveys have focused on awareness of specific alcohol policies, or attitudes to specific alcohol policy options, within a country or smaller region. There have also been cross-national surveys of the state of national-level alcohol policies (Anderson \& Baumberg, 2006; International Center for Alcohol Policies, 2002; World Health Organization, 2004). To our knowledge, no studies have yet been conducted to assess the state of alcohol policy development and implementation in response to the WHO Global Strategy (World Health Organization, 2010). The web survey had a $71 \%$ response rate. A meta-analysis of response rates for 68 web- or internet-based surveys of the general public, reported in 49 studies, found a mean response rate of $39.6 \%$, indicating a substantially higher response rate in this web survey (Cook, Heath, \& Thompson, 2000).

Responses varied slightly by sector, with response rates of $50 \%$ for respondents in the government sector, $68 \%$ among academics and researchers, and 86\% among respondents from NGOs/CBOs. The lower response rate for academics and researchers, compared to NGO/CBO respondents, could partly be explained by the fact that some academics were away on sabbatical leave, and, in one case, it was discovered that a researcher was ill. One government employee indicated that he had trouble accessing the web survey on his computer at work.

The increase in the ranking of Leadership in 2011, as compared to 2006, probably reflects the increased attention given to alcohol issues by various politicians post-2009. In contrast, the drop in the ranking of the mean rating on Marketing probably reflects the pervasive marketing of alcohol in the media, aimed at selling more products and responding to increased competition for market share. The drop in the ranking of the mean rating on Availability, in turn, probably reflects perceptions that there are an increasing number of unlicensed liquor outlets selling alcohol at all hours of the night and day, and is also probably a response to the delays in finalising legislation on liquor trading in certain provinces. The total (average) rating in 2011 of 35\%, while up by 11 percentage points in comparison to 2006, still indicates that much work remains to be done in terms of alcohol policy development and implementation in South Africa. Areas singled out as particularly in need of attention included Marketing, Availability, Informal alcohol, and Financing.

The NAPSC was found to be easy to use, and it has the potential to stimulate discussions within a country, particularly where there are likely to be differences between sectors. Such differences were identified, for example, between respondents from the government and other sectors on the issue of Leadership, awareness and commitment. The consistent findings across respondents (and sectors) of improvement in alcohol policy development and implementation between 2006 and 2011 suggest that the NAPSC can be used to monitor changes over time, something that is potentially useful for the World Health Organization and for individual countries.

In principle, the NAPSC could also be used to facilitate comparisons across countries, though differences between countries may be caused in part by variations in the degree to which members of different societies tend to be critical of government policy and implementation. The NAPSC focuses on formal alcohol (control) polices and does not includes a measure of what Karlsson and Österberg (2001) term "informal alcohol control," something which varies a lot between countries and which could also have an impact on the kinds of alcohol policies that are implemented, how they are enforced, and their effectiveness.

\section{Limitations}

The response categories for the NAPSC were determined by one person, and it could be argued that it would have been preferable to determine them though a consensus process involving an international panel of alcohol policy researchers. Some of the items comprised multiple concepts, and it is likely that some respondents found it difficult to give average scores for these items, thus bringing into question the validity of some of the ratings. The instrument also relies on self-ratings, and not all respondents will interpret the rating scales in exactly the same way. The choice of respondents in completing an instrument such as the NAPSC is crucial, as they need to have a broad understanding of alcohol policy. Not all respondents are likely to be equally well-versed in important policy details such as physical availability of alcohol and pricing policies. Such experts might be difficult to locate in some countries. However, with the sample size in this study, these issues are probably less critical. In some countries, some or all alcohol policies are determined at a level lower than the national level; in such countries, it might be preferable to ask respondents to complete the survey at a sub-national level.

A further limitation of the NAPSC is that each item is given an equal weight, when in fact some policy options are likely to be more effective than others and might need to be given a greater weight (Chisholm, Doran, Shibuya, \& Rehm, 2006; Karlsson \& Österberg, 2001). With regard to the comparison of ratings at two points in time, it is possible that asking about the later time period (2011) first may have biased the responses for the earlier time period (2006), to show progress. This could be addressed either by including only one time period, or by varying the order of the time periods from item to item.

It can, furthermore, also be argued that the NAPSC only indirectly measures what is actually going on at the local level with regard to alcohol policy implementation, and that it fails to address questions such as "Are people in different areas able to buy alcohol outside of trading hours?", "What is the price they are paying for alcohol on the streets?", and "How are they being reached by alcohol marketing?" This important data is complementary to the kind of macro-level information obtained by the NAPSC, and can be found in 
the International Alcohol Control (IAC) Study (Casswell et al., 2012), which is now being rolled-out in South Africa and other countries.

\section{Future research}

It is recommended that the NAPSC be refined and subjected to further evaluation. Among other things, it is recommended that (1) research be conducted to assess the reliability of the NAPSC among the same respondents at different time periods; (2) items with several concepts be divided into sub-items and the utility of assessing each subitem versus a composite item be compared; (3) policies and their implementation be measured separately; (4) research be conducted to formally validate the key informant ratings against external empirical measures of the existence of particular alcohol policies (e.g., via legislation) and their implementation (e.g., via newspaper reports and testimony of government officials, NGO workers and academics); (5) a measure for assessing the confidence of respondents' ratings on each item be added; (6) the web-based approach to completing the NAPSC be compared with an exercise where the survey is presented to a group of experts in a face-to-face setting and a consensus position is sought; and (7) further research be conducted to assess the utility of the NAPSC in different countries and in transnational studies.

\section{Conclusions}

This study broadly speaks to the need for the designers of global strategies to bear in mind how these strategies' implementation could be measured. The NAPSC can be used to promote discussions around the development and implementation of national alcohol strategies and will hopefully also contribute to the development of better alcohol policy measurement tools.

\section{Acknowledgements}

Hendra van Zyl, Head of the MRC Web \& Media Technologies Division, is thanked for her assistance in designing and implementing the web survey and ensuring the timely transmission of data from study participants in a way that ensured anonymity.

\section{References}

Anderson, P., \& Baumberg, B. (2006). Alcohol in Europe: A public health perspective. London, United Kingdom: Institute of Alcohol Studies.

Anderson, P., \& Lehto, J. (1995). Evaluation and monitoring of action on alcohol. Copenhagen, Denmark: World Health Organization Regional Office for Europe.

Babor, T., Caetano, R., Casswell, S., Edwards, G., Giesbrecht N., . . . Rossow, I. (2010). Alcohol: No ordinary commodity: Research and public policy. New York, NY, United States: Oxford University Press.

Brand, D. A., Saisana, M., Rynn, L. A., Pennoni, F., \& Lowenfels, A. B. (2007). Comparative analysis of alcohol control policies in 30 countries. PLOS Medicine, 4, 752-759.

Casswell, S., Meier, P., MacKintosch, A. M., Brown, A., Hastings, G., Thamarangsi, T., . . . You, R. Q. (2012). The International Alcohol Control (IAC) Study Evaluating the impact of alcohol policies. Alcoholism: Clinical \& Experimental Research, 36, 1462-1467. doi:10.1111/j.1530-0277.2012.01738.x

Chisholm, D., Doran, C., Shibuya, K., \& Rehm, J. (2006). Comparative cost-effectiveness of policy instruments for reducing the global burden of alcohol, tobacco and illicit drug use. Drug Alcohol Review, 25, 553-565.

Cook, C., Heath, F., \& Thompson, R. L. (2000). A metaanalysis of response rates in web- or internet-based surveys. Educational \& Psychological Measurement, 60, 821-836.

Davies, P., \& Walsh, B. (1983). Alcohol problems and alcohol control in Europe. London, United Kingdom: Croom Helm.

Department of Social Development. (2011). Ethekwini resolution on Second Biennial Substance Abuse Summit, 25 March 2011. Pretoria, South Africa: Author.

Hilton, M., \& Johnstone, B. M. (1988). International trends in alcohol consumption: A report on a symposium. Contemporary Drug Problems, 15, 685-716.

International Center for Alcohol Policies. (2002). Alcohol policy through partnership: Is the glass half-empty or half-full. Washington, DC, United States: Author.

Karlsson, T., \& Österberg, E. (2001). A scale of formal alcohol control policy in 15 European countries. Nordisk Ălkohol \& Narkotikatidskrift (English Supplement), 18, 117-131.

Parry, C. D. H. (2010). Alcohol policy in South Africa: A review of policy development processes between 1994 and 2009. Addiction, 105, 1340-1345.

World Health Organization. (2004). Global status report on alcohol policy. Geneva, Switzerland: Department of Mental Health \& Substance Abuse, World Health Organization.

World Health Organization. (2010). Global Strategy to reduce the harmful use of alcohol. Geneva, Switzerland: Author.

World Health Organization. (2011). Global status report on alcohol and health. Geneva, Switzerland: Author. 


\section{Appendix 1: Example of two items on the NAPSC}

\section{Item 1 on the NAPSC:}

Please give a rating between "1" and " 10 " for each of the 12 policy areas indicated to express your opinion of where South Africa is at currently ("2011") and then for each item (using your rating of the current situation as a reference) indicate where what you believe the situation was roughly five years ago (in 2006). Definitions of the endpoints (Very low (1) and very high (10) for ratings for each of the 12 areas have been provided.

1) National alcohol strategy

Very low (1) - No national policy or very fragmented

Very high (10) - Well set out, stand-alone policy with short-, medium- and long-term measurable objectives based on good evidence

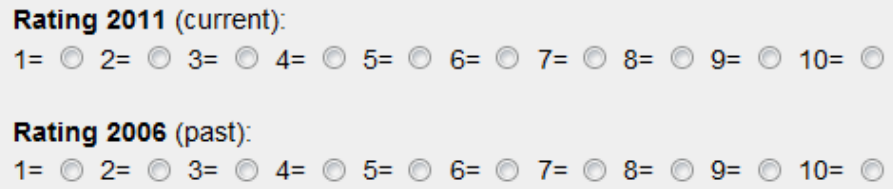

\section{Item 7 on the NAPSC:}

7) Drink-driving policies \& counter measures

Very low (1) - High (e.g. $1.0 \mathrm{~g}$ alcohol/100 ml of blood and higher) or no maximum BAC levels for drinking \& driving. Poor enforcement of drink-driving laws with little support from judicial system. No intervention programmes (mandatory drivereducation, counselling \& where appropriate treatment) for persons found guilty of drink driving offenses. No public awareness campaigns regarding dangers of drinking \& driving. Few public transport options for persons drinking away from where they live.

High (9) - Clear plan for addressing drink-driving that is adequately implemented (including enforced where required). Frequent random breath checks \& sobriety checkpoints; administrative license suspension for persons found to have BAC levels above those allowable. Backed up by a supportive law enforcement \& judicial system. Effective interventions for initial offenders (including license suspension \& need to complete an intervention programme held over several weeks, becoming more stringent for repeat offenses). Supported by good public awareness campaigns \& availability of alternative public transport. Having a low allowable BAC levels ( 0.02 to less than $0.05 \mathrm{~g} / 100 \mathrm{ml}$, especially for professional \& novice drivers) that are well enforced.

Very high (10) - No drinking and driving allowed (e.g. drivers not allowed to have BAC to exceed 0.00 (or 0.02 to allow for medicines).

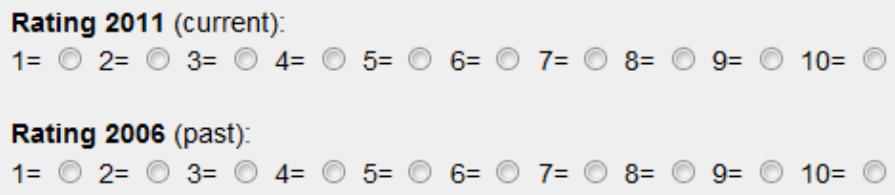

